\title{
Bioaerosols in the Barcelona subway system
}

\author{
X. Triadó-Margarit ${ }^{1}$ \\ M. Veillette ${ }^{2}$ \\ C. Duchaine ${ }^{2}$ \\ M. Talbot $^{2}$ | F. Amato ${ }^{3}$ | \\ M. C. Minguillón ${ }^{3}$ | V. Martins ${ }^{3}$ | E. de Miguel ${ }^{4}$ | E. O. Casamayor ${ }^{1}$ | T. Moreno ${ }^{3}$
}

${ }^{1}$ Centre for Advanced Studies of Blanes (CEAB), Spanish Research Council (CSIC), Blanes, Spain

${ }^{2}$ Centre de Recherche de l'Institut Universitaire de Cardiologie et de Pneumologie de Québec, Québec, QC, Canada

${ }^{3}$ Institute for Environmental Assessment and Water Research (IDAEA-CSIC), Barcelona, Spain

${ }^{4}$ Transports Metropolitans de Barcelona, TMB Santa Eulalia, Barcelona, Spain

\section{Correspondence}

Teresa Moreno, Institute for Environmental Assessment and Water Research (IDAEA-

CSIC), Barcelona, Spain.

Email: teresa.moreno@idaea.csic.es

Funding information

Spanish Ministry of Economy and

Competitiveness (MINECO); FEDER, Grant/

Award Number: METRO CGL2012-33066

and BRIDGES CGL2015-69043-P; European

Union Seventh Framework Programme,

Grant/Award Number: FP7/2007-2013;

Marie Curie ITN, Grant/Award Number:

315760 HEXACOMM; IMPROVE LIFE

Project, Grant/Award Number: LIFE13 ENV/

ES/000263

\begin{abstract}
Subway systems worldwide transport more than 100 million people daily; therefore, air quality on station platforms and inside trains is an important urban air pollution issue. We examined the microbiological composition and abundance in space and time of bioaerosols collected in the Barcelona subway system during a cold period. Quantitative PCR was used to quantify total bacteria, Aspergillus fumigatus, influenza $A$ and $B$, and rhinoviruses. Multitag 454 pyrosequencing of the $16 \mathrm{~S}$ rRNA gene was used to assess bacterial community composition and biodiversity. The results showed low bioaerosol concentrations regarding the targeted microorganisms, although the bacterial bioburden was rather high $\left(10^{4}\right.$ bacteria $\left./ \mathrm{m}^{3}\right)$. Airborne bacterial communities presented a high degree of overlap among the different subway environments sampled (inside trains, platforms, and lobbies) and were dominated by a few widespread taxa, with Methylobacterium being the most abundant genus. Human-related microbiota in sequence dataset and ascribed to potentially pathogenic bacteria were found in low proportion (maximum values below $2 \%$ of sequence readings) and evenly detected. Hence, no important biological exposure marker was detected in any of the sampled environments. Overall, we found that commuters are not the main source of bioaerosols in the Barcelona subway system.
\end{abstract}

\section{KEYWORDS}

airborne bacterial community, bioaerosol, culture-independent approach, indoor air quality, indoor microbial ecology, subway system

\section{1 | INTRODUCTION}

Air quality in public places has become an issue of concern for public health. Consequently, with the increase in knowledge on health problems associated with air pollution, the interest in bioaerosols has also increased. Due to their microscopic size, bioaerosols are able to remain airborne for long periods of time and travel long distances (eg, $\left.{ }^{1,2}\right)$. Bioaerosols can also easily get into the human respiratory tract, and indeed, depending on their composition, a fraction of the microbial burden can account for infectious diseases and at high concentrations produce toxins or act as allergens. ${ }^{3}$ As current lifestyle implies that humans spend about $90 \%$ of their time in confined spaces, ${ }^{4}$ where air could be a significant transmission pathway, monitoring the air quality of indoor environments is of major interest. ${ }^{5}$ However, despite its undeniable impact from the point of view of public health, there is comparatively little knowledge of the indoor air microbiome from public places, even if these spaces are usually very busy and can therefore be key locations for the transmission of pathogens. ${ }^{6}$

Subway stations are underground environments within a confined space and are sometimes poorly ventilated and crowded conditions which are likely to negatively affect the air quality. ${ }^{7}$ Given the fact that nearly 200 cities around the world have subway networks which are 
used by more than 100 million people every day, ${ }^{8}$ there is surprisingly little information published on subway aerial microbiological burdens $\left(e g,{ }^{6,9}\right)$. Furthermore, many of the studies focusing on airborne microorganisms (bacteria and/or molds) from subway networks have used culture-dependent approaches. ${ }^{8,10}$ As most environmental microbes are not currently cultured by such approaches, ${ }^{11}$ only a small fraction of the actual microbial content (generally $<1 \%$ ) can be identified with these techniques. Consequently, culture-independent approaches are needed for a better identification of airborne microorganisms. It is, however, clear enough from the research carried out so far that some studies have pinpointed Cladosporium, Penicillium, and Aspergillus genera (a set of environmental molds) as having high prevalence in some subway air samples. ${ }^{12-14}$ On the other hand, new studies on the characterization of the urban environment by means of a metagenomicbased approach (focused on the microbiome's analysis from surfaces) are currently in progress. ${ }^{15,16}$

In this study, we examine bioaerosols collected in the Barcelona subway system during an intensive monitoring campaign carried out over four months (November 2013-February 2014) inside trains, on platforms, and in lobbies. The system in Barcelona absorbs around $50 \%$ of the urban commuting load, transporting 1.25 million commuters on workdays. ${ }^{17}$ Trains are equipped with air-conditioning (AC) systems that when operating have a positive effect on the air quality inside the carriages by decreasing the concentrations of coarse particles $\left(\mathrm{PM}_{10}{ }^{18}\right)$. Air quality studies in the Barcelona subway network have to date focused on other aspects related to the air quality of the Barcelona subway system, such as factors controlling particulate matter concentrations and composition, ${ }^{17-21}$ so that our study was offering something new in attempting to characterize bioaerosols. In particular, we focused on the evaluation of microbiological composition and bioaerosol abundance in space and time using molecular methods such as quantitative real-time PCR on DNA (and cDNA) to quantify total bacteria, the fungus Aspergillus fumigatus, and the viruses influenza $A$ and $B$, and rhinoviruses. In addition, we carried out a pyrosequencing analysis of the $16 \mathrm{~S}$ rRNA gene to assess bacterial biodiversity.

\section{2 | METHODS}

\section{1 | Sampling site}

Trains from a total of six lines and four subway stations from the metropolitan underground network of Barcelona (TMB), Spain, were selected to take air samples during the 2013-2014 winter season. The Barcelona subway system comprises eight lines built progressively since 1924 to 2016 . The system carries around 376 million passengers a year and is chosen by about $50 \%$ of the urban population as their mode of public transport in the city. The platforms have a specific ventilation system that introduces outdoor air to renew the air throughout lateral ventilation outlets across the platform and extracts the aged air through a vertical well. In addition, a ventilation system operates in the tunnels where vertical wells introduce outdoor air into the tunnel and remove underground air toward the

\section{Practical Implications}

- With the increase in knowledge on health problems associated with air pollution, air quality in public places has become an issue of concern for public health. Hence, the interest in bioaerosols (the fraction of aerosols of biological origin) has increased, as they may have infectious, allergenic, or toxic properties. We examined for the first time the bioaerosols (including a set of target pathogenic microorganisms-Aspergillus fumigatus, influenza viruses, and rhinoviruses), inside trains and on platforms and lobbies, during a monitoring campaign within the Barcelona subway system. Low bioaerosol concentrations were found regarding the targeted microorganisms, commuters were not the main source of bioaerosols, and potentially pathogenic bacteria were found in low proportion. Thus, the study shows no major biological exposure evidence for commuters.

surface. All trains are operated using a rigid overhead catenary electric power supply and run from 05:00 h until midnight every day, with a frequency between 2 and $15 \mathrm{~min}$, depending on the day (weekend or weekday), subway line, and time of day. Trains from all lines are equipped with an efficient air-conditioning system (with windows impossible to open) that works continuously throughout the year to maintain a comfortable temperature, but with higher intensity during the summer period. Each of the four stations has a different architectural design, and it was chosen to obtain a wider range of sample characteristics, including a station with open double rail track (Santa Coloma), double track separated by a wall in the station (Joanic), single rail track (Tetuan), and a new station with platform screen doors (PSD's) separating the single rail track from the platform (Llefià). Over a four months period extending from November 18, 2013 to February 11, 2014, a total of 54 air samples (six each time) were collected at an average interval of one week except during the holiday season (late December/early January) when no sampling was performed.

Thus, for each selected date (nine series in total), two samples were taken inside trains (indicating whether AC was functioning or not), two samples at the boarding platform and two at the ticket office (lobby). Measurements inside the trains were performed in the middle of the central carriage of the train along the whole length of the line uninterruptedly with and without air-conditioning. The $\mathrm{AC}$ system has filters that are currently replaced monthly according to the manufactures instructions. The total duration of the trip depended on the length of the line and ranged from 45 to $90 \mathrm{~min}$. A manual record of the time when train doors opened and closed was taken, with an average time of $30 \mathrm{~s}$ for doors being opened at each station. To minimize the possible effect of major differences in passenger numbers on the results, all journeys were performed at the same time of the day (starting at 10:00 am), avoiding rush hour but 
when a moderately high number of travelers were present. Similarly, when comparing trips with and without $A C$, the samples were collected in the same train, traveling one way with $A C$ and making the return journey without $A C$. In addition, nine fields blanks, one for each collection day, were used to determine the level of baseline contamination.

Environmental conditions were recorded (temperature, relative humidity) while sampling at platforms and inside trains, with values showing low variability. The ranges were as follows: $21-24^{\circ} \mathrm{C}$ (temperature) and $32-38 \%(\mathrm{RH})$ were recorded on platforms, and $21-23^{\circ} \mathrm{C}$ (up to $26^{\circ} \mathrm{C}$ without air-conditioning) and $33-57 \% \mathrm{RH}$ inside the trains. Differences in temperature and $\mathrm{RH}$ when measuring with and without air-conditioning inside the same train varied from 0.5 to $2^{\circ} \mathrm{C}$ and 1 to $3 \%$, respectively.

\section{2 | Sampling and sample preparation}

Samples were collected at $1 \mathrm{~m}$ above the ground using a Coriolis ${ }^{\circledR} \mu$ (Bertin Technologies, Montigny-le-Bretonneux, France), in $15 \mathrm{~mL}$ of PBS (phosphate-buffered solution), at a rate of 200 liters of air/min for $10 \mathrm{~min}$. Samples were kept in a portable cold storage bag with ice packs and sent to the Barcelona laboratory on the same day, except on February 11 when they were shipped the next day. The collection fluid was divided into three aliquots (triplicate) of $1.5 \mathrm{~mL}$ each, which were centrifuged (10 min, $10000 \mathrm{rpm}$ ). Pellets were then stored at $-80^{\circ} \mathrm{C}$ until shipment to the Canada laboratory (Quebec City, Canada, CRIUCPQ).

RNA extraction was performed after the samples were pre-filtered (sterile units of $45 \mu \mathrm{m}$ pore size), and the remaining collection liquid was processed through a Microcon-30 kDa centrifugal filter unit column (MRCFOR030, EDM Millipore, Darmstadt, Germany). Volumes ranging from 100 to $140 \mu \mathrm{L}$ were used to perform the extraction using the Qiagen Viral RNA QIAamp ${ }^{\circledR}$. Extracted RNA was eluted in $50 \mu \mathrm{L}$ of elution buffer and contaminating DNA removed from RNA samples by treating with the TURBO DNA-free ${ }^{T M}$ kit (Ambion, Austin, TX). The manipulation was performed according to the manufacturer's instructions.

CDNA synthesis was carried out using iScript cDNA Synthesis ${ }^{\circledR}$ kit (Bio-Rad Laboratories, Hercules, CA, USA), and $15 \mu \mathrm{L}$ of the 50 $\mu \mathrm{L}$ RNA extracted previously was used for the RT reaction. The final reaction mixture $(20 \mu \mathrm{L})$ was as follows: $15 \mu \mathrm{L}$ of sample, $4 \mu \mathrm{L}$ of the iScript $5 \times$ reaction mix, and $1 \mu \mathrm{L}$ of the iScript reverse transcriptase. The PCR reaction was performed according to the manufacturer's recommendations. The tubes containing the cDNA were then stored at $-80^{\circ} \mathrm{C}$ until shipment to the laboratory (Quebec, Canada, CRIUCPQ).

\section{3 | Transportation and storage}

The samples including 189 pellets of $1.5 \mathrm{~mL}$ samples prepared for DNA extraction, the corresponding RNA extracts (without DNA) (30$35 \mu \mathrm{L} /$ tube) and the cDNA ( $20 \mu \mathrm{L} /$ tube), were shipped on dry ice. Upon arrival at the Quebec laboratory, they were immediately transferred to $-80^{\circ} \mathrm{C}$ freezer, and so, until their analysis.

\subsection{Extraction and purification total DNA}

The DNA of the air samples was extracted using the Qiagen commercial kit (Qiagen, Mississauga, ON, Canada), QIAamp ${ }^{\circledR}$ DNA Mini Kit, according to the protocol established by the manufacturer, but modified to include a lysozyme digestion. The purified DNA was eluted in $200 \mu \mathrm{L}$ of $\mathrm{AE}$ buffer, and samples were stored at $-20^{\circ} \mathrm{C}$ until use.

\section{5 | Preparation of standard curves}

DNA from E. coli (\#ATCC: 35218) and A. fumigatus was extracted using the Qiagen commercial kit (Qiagen, Mississauga, ON, Canada), QIAamp ${ }^{\circledR}$ DNA Mini kit according to the manufacturer's recommendations, except a $180-\mu \mathrm{L}$ ATL buffer was used instead of a $200-\mu \mathrm{L} A \mathrm{~L}$ buffer. In the case of the viruses, previously prepared plasmid DNA containing PCR targets was used. DNA (genomic of plasmids) per tube was assayed in order to calculate the volume to be suspended in TE buffer for $5 \times 10^{5}$ copies of genome/ $\mu \mathrm{L}$. Then, $10 \mu \mathrm{L}$ aliquots were prepared. The results of the concentration of total bacteria have been expressed in terms of E. coli.

\section{6 | Quantitative PCR (qPCR)}

PCR amplifications were performed using a thermal cycler CFX384 Touch $^{\text {TM }}$ real-time PCR System (Bio-Rad Laboratories, Mississauga, ON, Canada). Quantification of total bacteria, A. fumigatus and the three viruses, was performed using the different primer pairs and probes listed in Table S1. The composition of the reaction mixtures and the various amplification programs used are presented in Tables S2 and S3. To quantify the DNA, serial dilutions $\left(10^{6}\right.$ to $10^{\circ}$ genome/ $\mu \mathrm{L}$ ) of E. coli and A. fumigatus genomic DNA, as well as plasmid DNA containing target site from each virus, were used to make standard curves. In all cases, data were acquired with the CFX Manager ${ }^{\text {TM }}$ (Bio-Rad Laboratories, Version 3.0.1224.1015) software which automatically determined threshold values. Negative controls (NTC) were also included in each PCR assay. All samples including controls were analyzed in duplicate. The concentrations obtained for the NTC were subtracted from each series. The chosen protocol for bioaerosols quantification has been used in different type of environments with no inhibition observed..$^{22-25}$

\section{7 | DNA pyrosequencing}

Air samples were pooled according to their location for the same stations, train line, and the presence of not of air-conditioning. A total of 20 samples were then used for sequencing. Bacterial tagencoded FLX amplicon pyrosequencing (bTEFAP) was performed at the Plateforme d'analyses génomiques of the Institut de Biologie Intégrative et des Systèmes (IBIS, Université Laval, http://www.ibis. ulaval.ca/?pg=sequencage) on a Roche $454 \mathrm{FLX}$ instrument with titanium reagents following manufacturer's procedures. The variable regions V6-V8 of the $16 \mathrm{~S}$ rRNA gene were amplified with the primers described previously. ${ }^{26}$ 


\section{8 | DNA sequence and statistical analyses}

bTEFAP sequences provided after Roche standard procedures of both quality checking and reads denoising were processed using the UPARSE pipeline ${ }^{27}$ version usearch8.0.1623_i86osx32. Sequences were trimmed in length, $250 \mathrm{pb}$, which substantially reduce the error rate. Approximately $53 \%$ of the original reads (102 397) passed the filter (corresponding to 54493 reads). The sequences were then clustered into operational taxonomic units (OTUs) using a cutoff of $0.03 \%$ as identity threshold and, in addition, analyzed to discard chimeric reads by means of a double strategy using UCHIME: de novo and reference-based chimera filtering step against "Gold" reference database available for ChimeraSlayer. Unique sequences (ie, singletons) were removed. Overall, 49752 sequencing reads could be assigned to some of the OTU representative sequences, which were then parsed to create an OTU table. A total of 136 OTUs were subsequently analyzed, and an average sequence depth of 2488 sequences per sample was obtained. In order to minimize effects of sampling effort on all OTU-based analyses, the original OTU table was average rarefied (based on 100 random subsamplings without replacement) to a depth of 1000 sequences per sample using the script multiple_rarefactions even_depth.py available in the Quantitative Insights Into Microbial Ecology toolkit-QIIME- ${ }^{28}$ Using these settings, a sample of the original dataset was discarded (corresponding to the lobby in Santa Coloma station), because had fewer sequences than the requested rarefaction depth. Taxonomic assignment was carried out with SINAAligner and classifier (version 1.2.11) using SILVA_119 reference database ${ }^{29,30}$ with the de-replicated version at $99 \%$ sequence similarity. Sequences original from bTEFAP representative OTUs were deposited in GenBank with accession numbers LT158063-LT158198.

All statistical analyses were run in the R environment (http:// www.r-project.org/). Community ecology-related parameters were calculated using the vegan package. ${ }^{31}$ Community similarities were represented by non-metri multidimensional scaling (metaMDS function) using Bray-Curtis dissimilarities after Hellinger standardization. ${ }^{32}$ Additionally, analyses of similarities (ANOSIM) were performed based on 1000 permutations to test for significant differences between a priori sampling units from the data matrix (ie, otu table), as the different compartments analyzed from the subway system (Inside trainwith air-condition or without-Lobby and Platform). The ANOSIM R statistic is based on the difference of mean ranks between groups and within groups and ranges from 0 (no separation) to 1 (complete separation). ${ }^{33}$ The multivariate homogeneity of group's dispersions (variances) was checked before proceeding with ANOSIM with the aim to discard heteroscedasticity among groups (permutest.betadisper function), and latterly used as a means of assessing the beta-diversity. Shannon indices and expected species richness were calculated using the functions diversity and rarefy (respectively); however, in the second case, the original OTU table was used to avoid its overestimation. Hypothesis contrast test was carried out with stats package; nonparametric tests were used when the assumptions for the parametric equivalents were not achieved. For the data descriptions showing the taxon composition, the taxonomic information related to the OTUs was summarized (at genus level when possible) by means of the summarize_taxa.py script available in QIIME. Venn diagram analysis was performed by means of the venn command available in Mothur. ${ }^{34}$ The isolation source category ascribed to the taxons was based on the habitat and ecology information available in "The Prokaryotes-A Handbook on the biology of bacteria," and further refined when aiming to determine its putative human origin using data available in Human Microbiome Project. ${ }^{35}$ All graphs were constructed with the ggplot2 package. $^{36}$

The presence of airborne potentially pathogenic bacteria in the bTEFAP dataset was analyzed by means of BLAST analyses against a de novo created database of 16 S rDNA sequences representing 275 bacterial species of both obligated pathogens and a number of opportunistic ones. The database used for searches was constructed as from the list of pathogenic species inventoried in a previous work ${ }^{37}$ and further refined by means of online searches of the ascribed taxa. For such analyses, just those phylotypes presenting sequence identity values above $98 \%$ were considered. In addition, the highest BLAST alignment coverage values were prioritized (threshold set at 99\%). As even identity values at $100 \%$ would need additional confirmation steps of such pathogenic capacity by studying specific virulence markers, the results here presented should be interpreted with caution and, thus, regarded as indicative of potentially pathogenic bacteria (ie, one would incorporate it to the concept of indicator species). The phylotypes adscribed to potential pathogenic bacteria were further classified in accordance with their host or pathogenic potential (obligated or opportunistic).

\section{3 | RESULTS}

\section{1 | Quantification of airborne target microorganisms}

\subsection{1 | Influenza A and B}

The viral load related to influenza on the subway indoor air was assessed by qPCR analyses. Influenza was detected in a number of samples: 30 of 54 in case of influenza A and 26 of 54 in case of influenza B were positives. Roughly, their frequency of detection was similar among different compartments analyzed. On average, the concentrations of influenza A were higher than those obtained for influenza $B$, being $2.66 \times 10^{2}$ genomes $/ \mathrm{m}^{3}$ and $1.82 \times 10^{2}$ genomes $/ \mathrm{m}^{3}$, respectively ( $t$-test, $P$-value $<.05)$. On the other hand, Kruskal-Wallis test confirmed that both, influenza $A$ and $B$, did not significantly vary among groups of samples (trains, platform or lobby) (Figure 1A), although concentrations seemed slightly lower in platforms and lobbies. We did not observe differences according to the setting of the AC system or between different stations (last data not shown).

Influenza activity as reported by Google flu trends (www.google. org/flutrends/) and data from Information System of Primary Health Care Services (SISAP) of the Catalan Institute of Health (ICS) for the Catalonia region in winter 2013-2014 showed that for this flu season, the peak of cases occurred during the sampling campaign (Figure S1). More in detail, we were able to detect significant differences among 
influenza concentrations between the two sampling periods of such winter campaign (Mann-Whitney $U$-test, $P$-value < .01) (Figure 1B). Here, a greater incidence of influenza before the winter holidays was observed.

\subsection{2 | Rhinovirus}

Rhinovirus was seldom detected during the studied period. The only quantifiable samples contained concentrations of $1.02 \times 10^{3}, 3.08 \times$ $10^{1}$, and $1.06 \times 10^{3}$ of rhinovirus genomes $/ \mathrm{m}^{3}$ of air. The first two concentrations came from air samples taken inside trains with $A C$, while the other came from the air of a train without AC.

\subsection{3 | Aspergillus fumigatus}

The quantification of $A$. fumigatus showed that only three of the 54 samples yielded concentration greater than the detection limit.
Indeed, a sample from a train with AC contained $1.52 \times 10^{2}$ of $A$. fumigatus genomes $/ \mathrm{m}^{3}$ of air; while on platform and lobby, they contained $8.12 \times 10^{2}$ and $1.78 \times 10^{2}$ of $A$. fumigatus genomes $/ \mathrm{m}^{3}$ of air, respectively.

\section{2 | Bacterial quantification and diversity}

Bacterial load was higher than the values reported by target microorganisms, as expected. The concentrations ranged from $1.07 \times 10^{3}$ to $3.29 \times 10^{6}$ equivalent $E$. coli genomes $/ \mathrm{m}^{3}$ of air, which correspond to an average concentration of $4.46 \times 10^{4}$ equivalent $E$. coli genomes/ $\mathrm{m}^{3}$ of air. Kruskal-Wallis test confirmed that there is no significant difference ( $P$-value $>.05)$ between groups of samples (train, platform, or lobby) (Figure 2) and also between different stations. In addition, total bacteria were not significantly different in relation to the setting of the air-condition system or sampling period (Mann-Whitney U-test, $P$-value $>$.05) (data not shown).
FIGURE 1 Comparison of concentrations of gene copies of specific markers for airborne influenza viruses determined by qPCR analyses from samples of Barcelona subway system ( $n=54)$. The panel A allows the comparison of concentration values between the different compartments analyzed, whereas panel B shows a comparison among different time periods
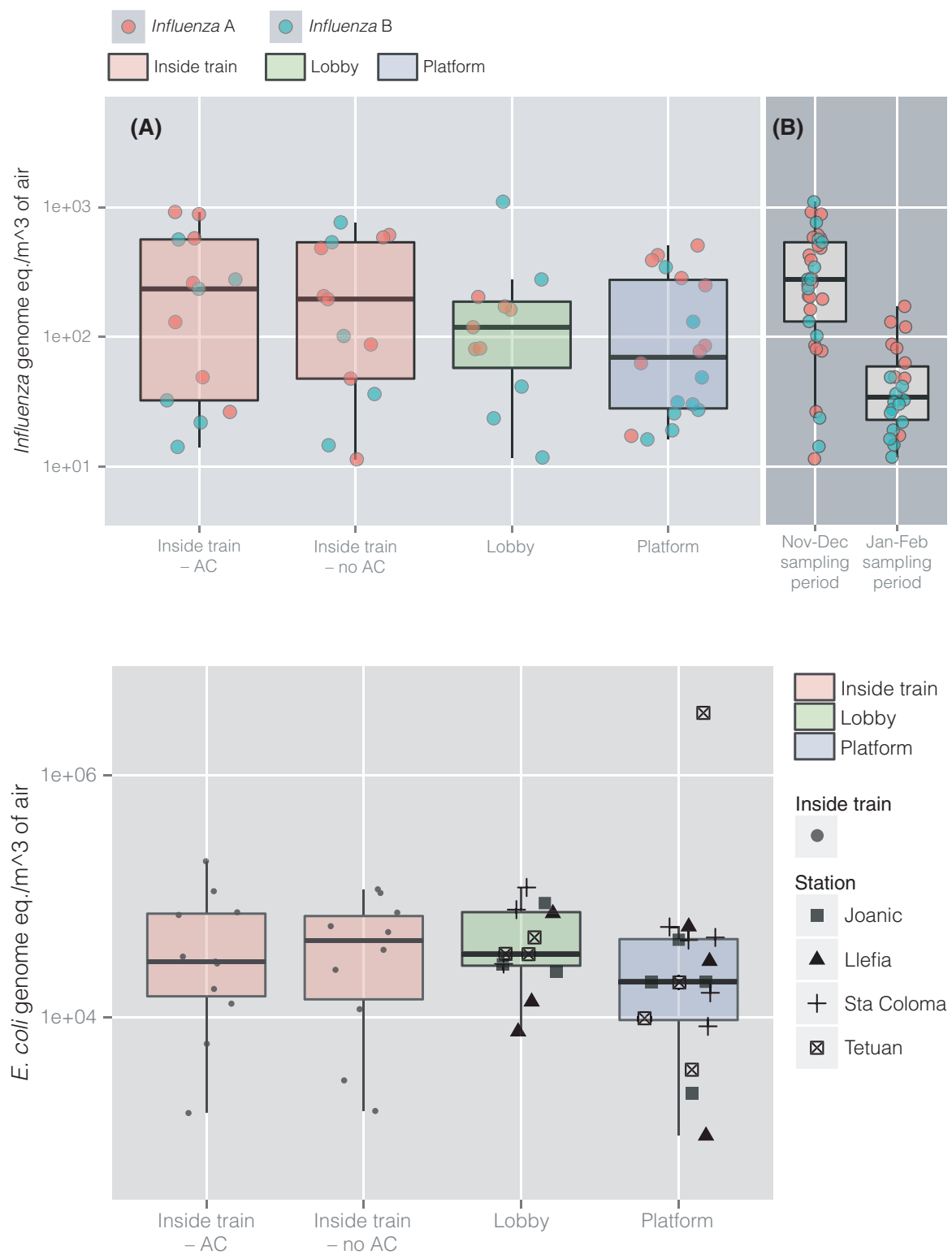

FIGURE 2 Concentrations of airborne Bacterial 16S rRNA gene copies determined by qPCR analyses for different compartments of the Barcelona subway system $(n=54)$ 


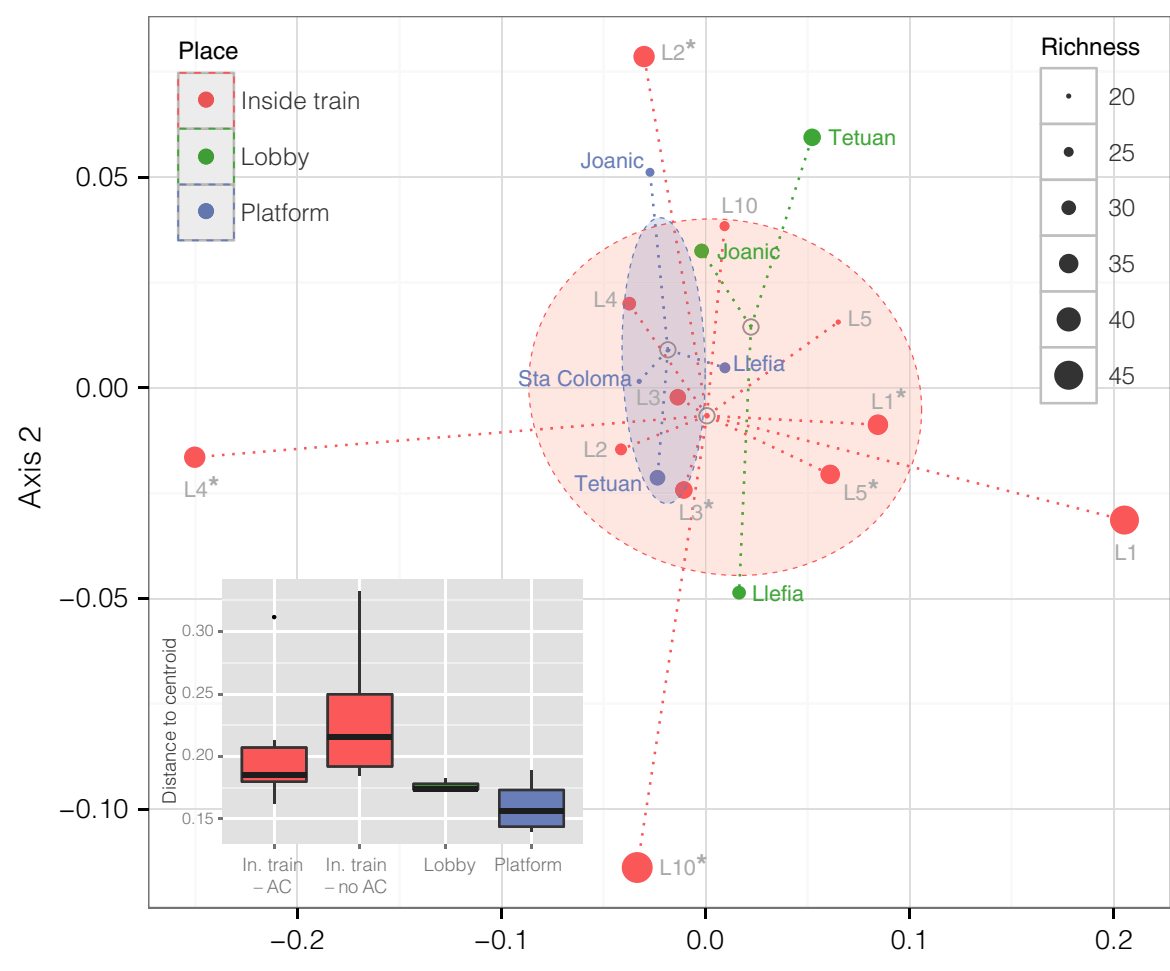

FIGURE 3 Non-metric multidimensional scaling (NMDS) ordination analysis of community similarities based on BrayCurtis dissimilarities. Colors are related to the place of origin of samples in the subway system, and point size is proportional to the OTU richness (set at 0.03 cutoff). Each point corresponds to the different sample types analyzed, from either different lines or stations, and indicated with specific labels; in addition, asterisk is indicating those samples taken from inside train without air-condition. Dotted lines join each sample with its corresponding group centroid. The ellipsoid areas are indicative of distribution (and dispersion) of each group on the ordination space. The inner panel contains boxplots of beta-diversity as distance to centroid for different groups of samples compared
Bacterial communities did not present significant differences in composition and structure among compartments (inside train-AC or non-AC-platform or lobby), as shown in NMDS ordination analysis based on Bray-Curtis dissimilarities (Figure 3). ANOSIM statistic further confirms the high degree of overlapping ( $R$ : -0.09635 significance: 0.887$).{ }^{38}$ In addition, the multivariate homogeneity of groups' dispersions confirmed no differences $(P$-value $=.112)$ (inner panel in Figure 3). The ecological diversity (Shannon index) and specific richness were rather low in comparison with other environmental sequence datasets-normally set at hundreds of species and above and $\mathrm{H}^{\prime}$ values around 3 or even higher (Figure 4). For instance, $\mathrm{H}$-values lower than 2 in all cases would indicate both low richness and high dominance of a small number of taxons. In addition, not significant differences were observed between compartments for these alpha-diversity indicators (ANOVA, P-value > 05 in both cases).

Regarding identified taxa, 19 of 87 (22\%) were found to overlap for the locations inside train, platform, or lobby (Figure 5A, Table S4), that is, they were ubiquitously detected, and so regarded as widespread taxa. Within this category, Methylobacterium, members of Chitinophagaceae, Bradyrhizobium, Paracoccus, and Sphingomonas were detected at high proportion and high frequency, that is, mean relative abundances $>0.5 \%$ and occurrence $>75 \%$ (Figure $5 B$ ). Genera related to human microbiota as Staphylococcus and Neisseria were detected at rather high proportion (mean relative abundance $0.5-1 \%$ ) but at lower frequencies (<50\%).

Among the two different air-conditioning settings tested (switch on or off) and locations within subway system, we did not find significant differences on the relative abundance of widespread-abundant-taxa
(AnOva, $P$-value $>$.05). Similarly, when considering all taxa putatively related to human microbiota (either commensal or potentially pathogenic), significant differences in mean relative abundances between the air-conditioning settings were not found ( $t$-test, $P$-value $>.05$; Figure S2). In addition, the frequency of such bacteria was similar between both groups. Thus, after the air-conditioning system was switched off, we did not observe higher occurrence of human-related bacteria.

\subsection{The presence of potentially pathogenic airborne bacteria}

Through BLAST analysis, we linked the 16S rRNA gene sequences to species or genera for which pathogenic strains (or varieties) have been previously reported (Table 1). Thus, they were regarded as an indicator of the presence of potentially pathogenic airborne bacteria. Overall, this group showed low mean relative abundances in the dataset, c. $0.25 \%$. The highest values were rather low, normally $<2 \%$. Interestingly, Staphylococcus epidermidis, which has been reported as an opportunistic pathogen ${ }^{39}$ (ie, just affecting immunocompromised patients in nosocomial infections), presented the highest abundance. Those potentially pathogenic bacteria, in most of cases, were evenly detected in the different sites analyzed.

\section{4 | DISCUSSION}

Despite the effect on human health of bioaerosols breathed in public transport systems being of broad research interest, only a small 


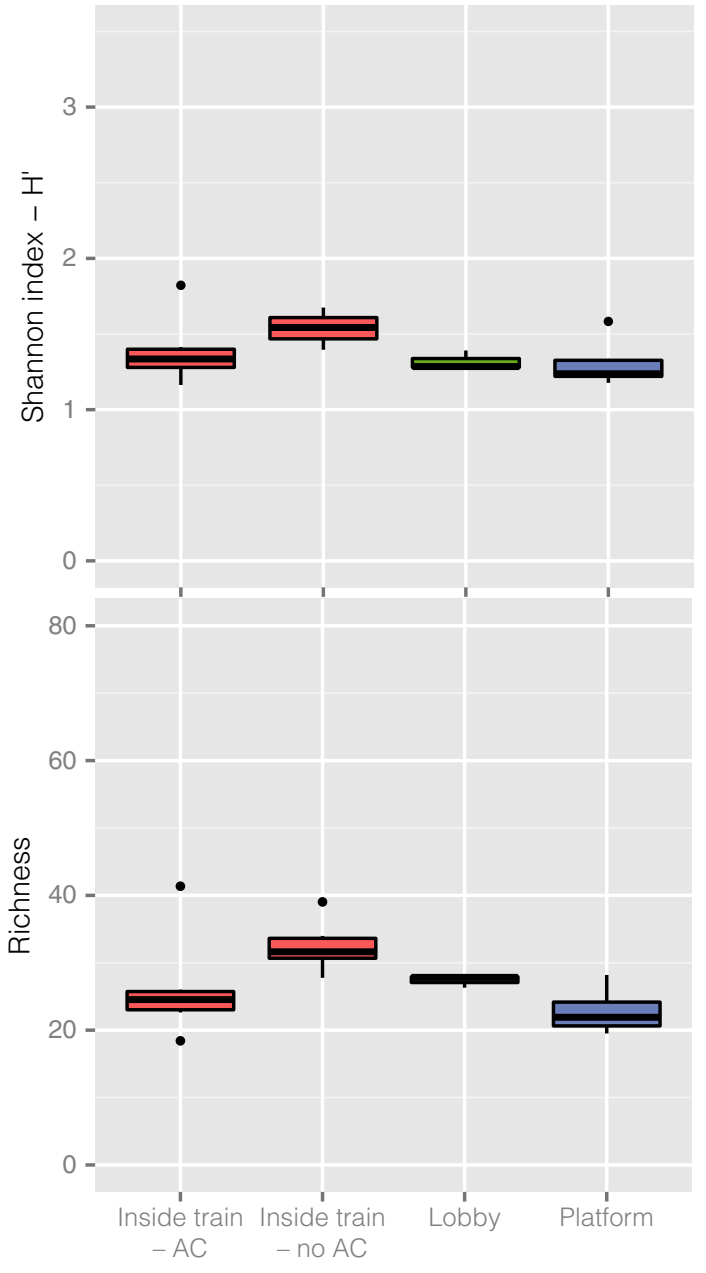

FIGURE 4 Boxplots of bacterial diversity (Shannon index, upper panel) and bacterial richness (as number of OTUs at 0.03 cutoff, lower panel) for different analyzed compartments in the Barcelona's subway system

number of studies have focused on bioaerosol exposure of citizens in subway stations $\left(\mathrm{eg}^{6,9}\right)$. Bioaerosol sampling in subway stations and trains requires several compromises related to the sampling method that needs to be portable, must collect the sample quickly, has to be robust, and require minimal manipulation. Sample $2 \mathrm{~m}^{3}$ of air for a short time has achieved an acceptable limit of detection but provides little information on the temporal variations of concentrations of bioaerosols. Repeated longitudinal sampling will provide a better picture of the presence of bioaerosols but will also be performed at high speed as it is expected that concentrations are low. We studied the microbiological air composition within the Barcelona subway system to improve knowledge on what bioaerosols commuters are daily exposed to and in which concentrations.

The results presented show low overall bioaerosols concentrations for the specific targeted microbial populations. However, total airborne bacteria averaged approximately $10^{4}$ bacteria $/ \mathrm{m}^{3}$, which is higher than values previously reported. ${ }^{8,40,41}$ For example, as previously studied in Seoul and Oslo stations, the average concentration of viable bacteria in the air was a few hundred CFU/m $\mathrm{m}^{3}{ }^{8,41}$ However, when microscopic counts were carried out, similar bacterial concentrations to ours were found, ${ }^{6}$ and it was shown that fluorescence microscopy and qPCR methods were comparable to each other. ${ }^{42}$ Thus, the discrepancy is most probably related to the use of molecular methods rather than cell culture as culture methods are known to underestimate real concentrations. ${ }^{43-45}$

We did not observe significant differences in total bacteria concentrations among the different track configuration included in this study. These results do not follow the trend observed for $\mathrm{PM}_{2.5}$ concentrations at the same sampling locations. Thus, previous studies described that the $\mathrm{PM}_{2.5}$ concentrations were lower inside trains, especially when $\mathrm{AC}$ was functioning. ${ }^{18}$ As the sources of airborne bacteria (outdoor air, humans) and $\mathrm{PM}_{2.5}$ (breaks and mechanical friction and wear) are different, the observed lack of correlation between both pollutants is not surprising.

The qPCR specific to influenza $A$ and $B$ virus yielded similar mean concentrations of the order of $10^{2}$ influenza genomes $/ \mathrm{m}^{3}$ of air (Figure 1A). This value would be 100 -fold lower, in case of influenza A, than other previously measured at indoors of different public places. ${ }^{46}$ No information was available in case of influenza B. In order to better interpret data and to determine whether subway users can be exposed during commuting to influenza virus, we performed a rough estimation of the potential inhaled dose during fall-winter studied period (November 2013-February 2014). Assuming a uniform airborne concentration (related to the mean value) of $2.66 \times 10^{2}$ genomes $/ \mathrm{m}^{3}$ of air and $1.82 \times 10^{2}$ genomes $/ \mathrm{m}^{3}$ of air for influenza $A$ and $B$, respectively, and an adult breathing rate of $20 \mathrm{~m}^{3} / \mathrm{day}^{46}$, we estimate the inhalation doses during exposures of $1 \mathrm{~h}$ to be $2.2 \times 10^{2}$ total viral particles in case of influenza A and $1.52 \times 10^{2}$ total viral particles for influenza B. Here, we assume that each genome equivalent represents one viral particle and that not all viral particles are necessarily infectious. Our assay did not test for viral infectivity, but one should consider that the ratio existing between infectious versus total influenza virus particles in laboratory stocks was established roughly as two orders of magnitude lower. ${ }^{47-49}$ Although it is not known whether this ratio could be extrapolated to environmental samples, one must take into account this loss in infectivity when aiming to estimate the infectious counts (eg, Tissue Culture Infectious Dose- $\mathrm{TCID}_{50}$ ). The presented value could be lower if considering that the most frequent average journey time in the Barcelona subway was previously reported to be 35 min. $^{17}$ Conversely, rhinoviruses presented a much lower frequency; they were just detected three times. In such case, the potential of commuters to be exposed was not demonstrated but further longitudinal sampling should be executed to better describe potential metro users exposure ${ }^{50}$ especially during the outbreak's period (typically in the fall).

Interestingly, influenza concentrations reported from November-December period were significantly higher compared to January-February. Nevertheless, data reporting influenza activity (from institutional entities of Catalonia's Government and from public Web facilities of Google Inc.) showed that the influenza peak occurred during the later period. Here, we assume that our methodological approach has low bias as qPCR primers sets were originally 
(A)

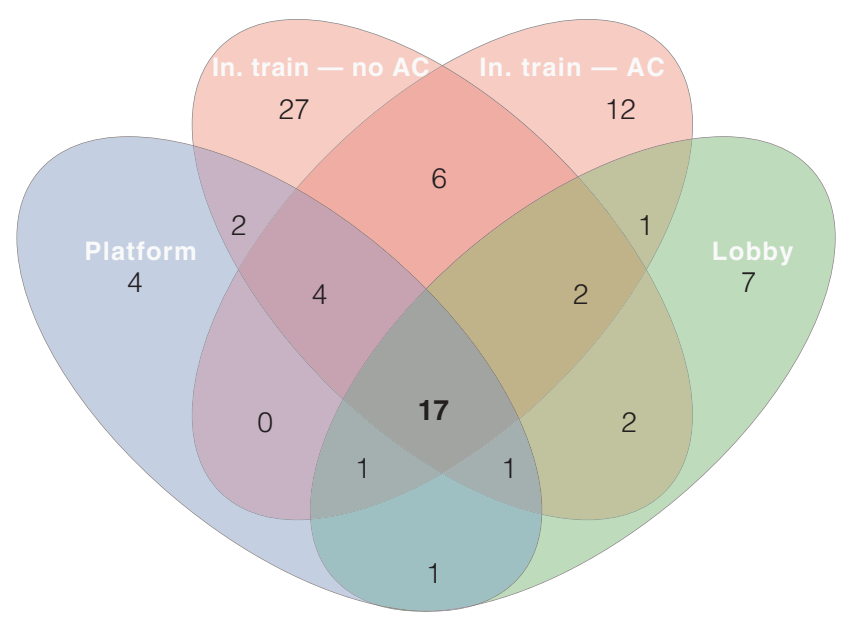

(B)

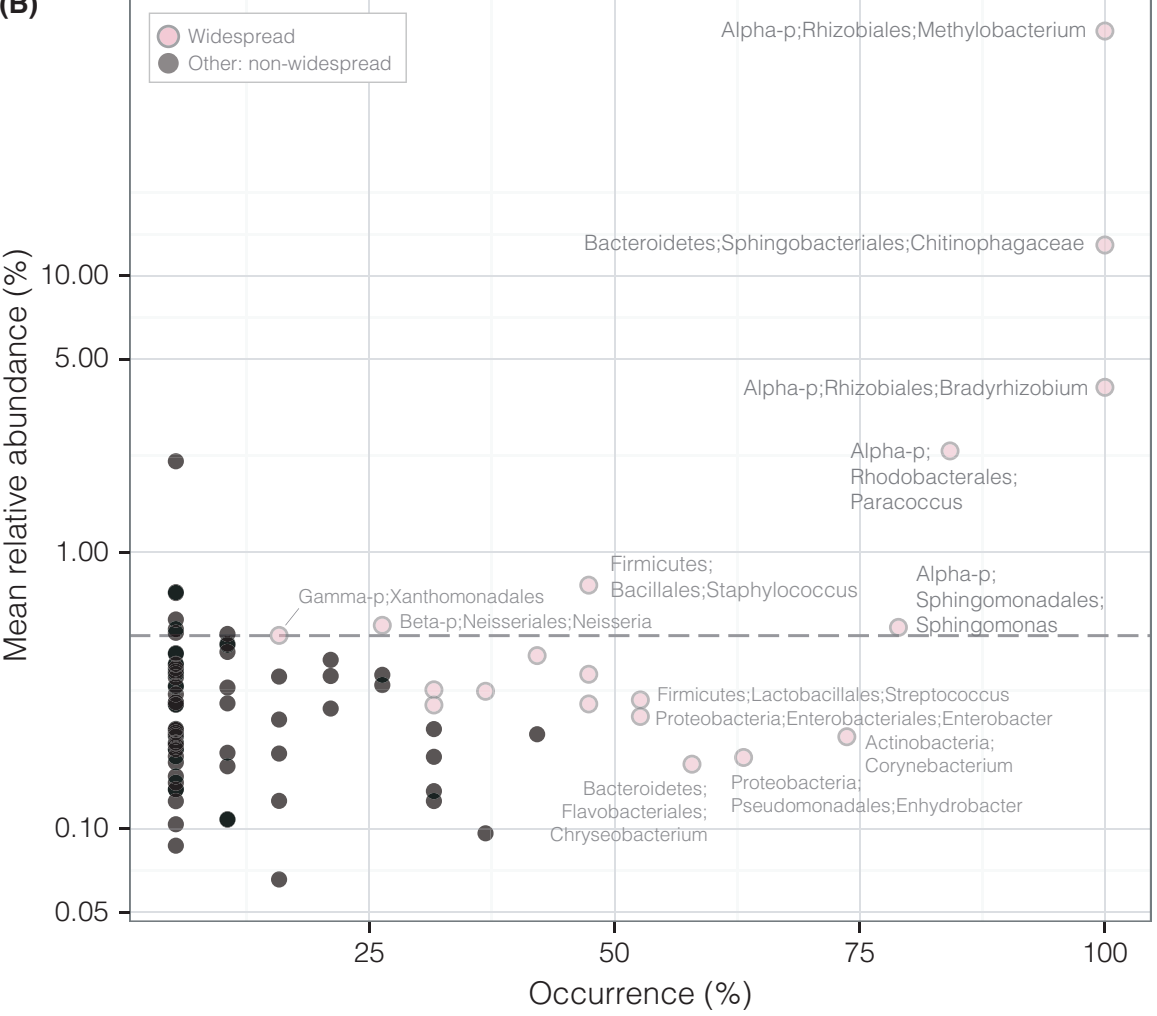

FIGURE 5 Venn diagram showing taxon overlap between different compartments in the Barcelona subway system (panel A). Mean relative abundances (where present) and occupancy relationships for bacterial taxons (panel B). The points are colored in accordance with their widespread character. The taxonomic labels for those taxa reporting either mean relative abundance values above $0.5 \%$ or occurrence above $50 \%$ are shown designed for universal detection of influenza (A and B), ${ }^{51}$ and therefore, we are reporting total concentrations from several influenza varieties and strains being in the aerosols. Thus, from this observation, a number of questions, which should be attended in further investigations with samplings along different flu outbreaks, arise. One might wonder if it is probable to obtain similar results in the future or if, instead, this is a kind of spurious and unique finding. If this happens regularly, then these data could give us some capacity to make predictions over oncoming flu outbreaks. In spite a rather high number of new questions, we have also observed during the flu peak that the influenza $A$ concentrations were significantly higher than those detected for influenza B (Welsh $t$-test, $P$-value <.01). This might be in accord with data reported by Public Health Agency of Catalonia (ASPCAT) where $99.7 \%$ of severe cases reported during the 2013-2014 campaign were related to influenza A (varieties H3, H3N2, and H1N1pmd09).

Regarding the quantitation of $A$. fumigatus, when detected, it was in average of $10^{2}$ per $\mathrm{m}^{3}$ of air. This is consistent with the results obtained by Bogomolova and Kirtsideli ${ }^{12}$ where the bacterial load of the St. Petersburg subway air was higher than the fungal one. Furthermore, once again, the concentrations obtained were much higher than what is usually found in the literature, again probably due to the same reasons previously stated in relation to the culture-independent methods. When comparing our data with other subways, it is important to consider the characteristics of the Barcelona subway system. All stations where samples were obtained were underground and have a ventilation system introducing outdoor air throughout lateral ventilation outlets across the platform and extracting air through a vertical well. 


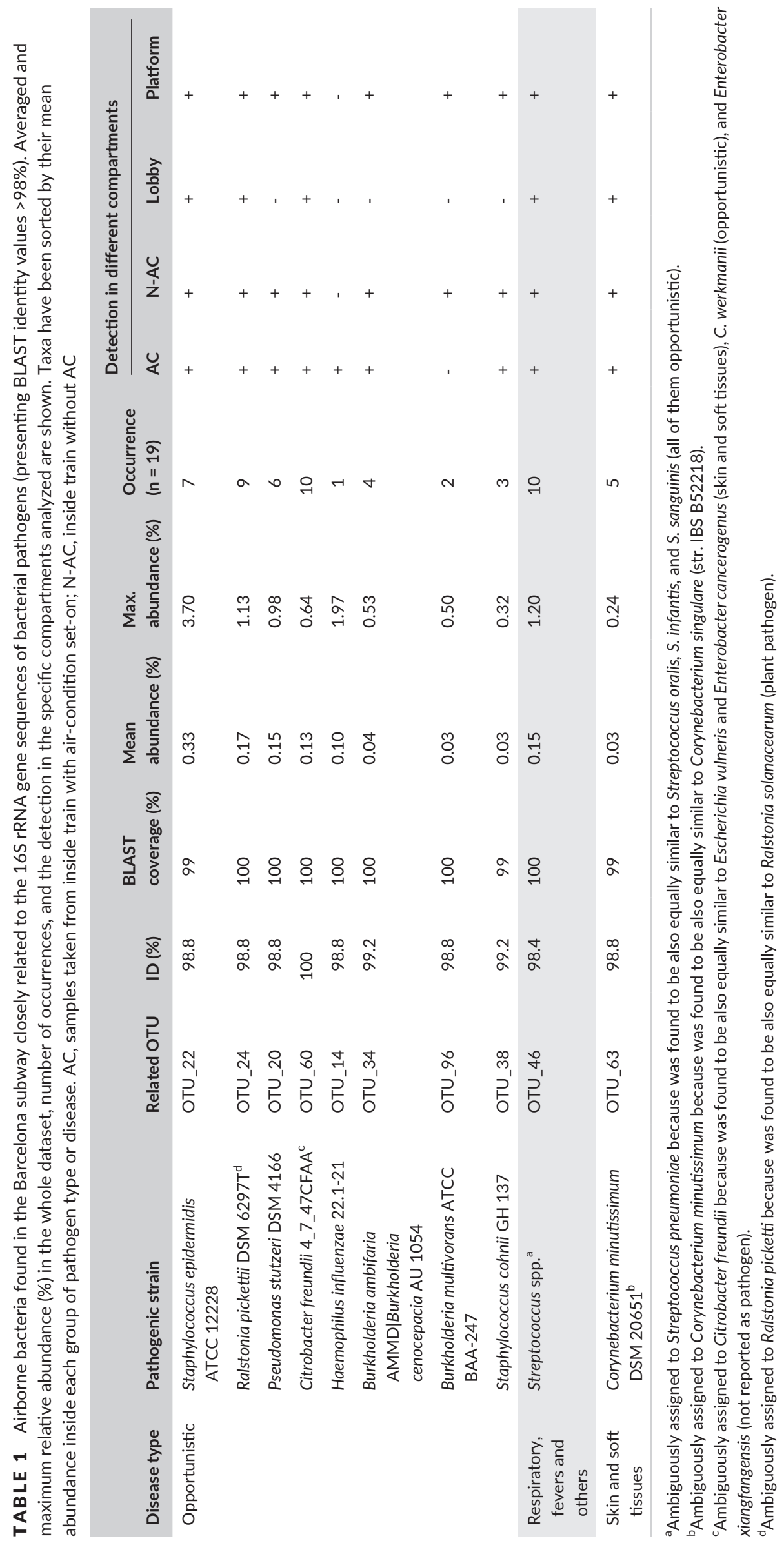


Furthermore, all trains are equipped with a filtered air-conditioning system and windows are kept closed. Although few samples in our study were positive (only three), no conclusions can be drawn regarding the absence of health risk. Indeed, the presence of certain pathogens such as A. fumigatus is considered unacceptable in indoor air and considering the absence of an exposure limits value to mold, the risk of allergic lung disease for certain categories of passengers at greater risk (immunocompromised, or elderly asthmatics) can exist. ${ }^{12}$ Nevertheless, considering that its origin is probably outdoor air, as it is one of the most ubiquitous of the airborne saprophytic fungi, ${ }^{52}$ we could conclude that the risk of exposure is not more elevated in the subway than in outdoors.

The analysis of airborne bacterial communities reveals that the commuters are not the main source generating bioaerosols in the subway system. Here, it is interesting to note that most recurrent and abundant taxa did not match to those regarded as human microbiota (neither commensal nor pathogenic). Nevertheless, our statement should be taken with care, as we lacked sampling during rush hours. As changes in microbial communities between peak and non-peak commuting hours were previously described and related to increases in skin-associated genera in peak samples, ${ }^{9}$ our study would be more representative of middle-to-low density periods. Thus, we may have underestimated bioaerosols generated from commuters and then also its relative abundance in the dataset. However, Adams et al. ${ }^{53}$ reported recently that a weak influence of human occupancy on microbial communities of built environments could exist, specifically from those well ventilated with a moderate occupancy. Actually, even in a high-traffic-influenced building, it was found that indoor air communities closely tracked those in outdoors. ${ }^{6,54}$ However, a higher proportion of human-associated bacterial genera were found in indoor air. ${ }^{54}$ Conversely, a previous study reached opposite conclusions, ${ }^{8}$ being anthropogenic sources the major contributors to airborne bacteria. However, a culture-dependent approach was used instead, and therefore, results are not comparable.

Airborne bacterial communities in Barcelona subway system were largely dominated by a limited number of taxa, where Methylobacterium was the most abundant. Moreover, some varieties of this genus have shown the ability to grow in the presence of particulate exhaust material (soot), and so, their possible role as biological monitors of vehicular pollution has been suggested. ${ }^{55}$ Indeed, it was found that Methylobacterium might grow with some of the polycyclic aromatic hydrocarbons (PAHs) and long-chain aliphatic hydrocarbons contained in such material. The subway systems are distinctive microenvironments where the concentration of pollutants from both the atmosphere and those generated internally can occur. ${ }^{7}$ Barcelona's subway aerosol is a complex mixture of compounds dominated by hematitic ferruginous particles sourced from the abrasion of wheels, rail tracks, and brakes, ${ }^{21}$ but also containing a high proportion of carbonaceous materials mainly derived from brake and catenary wear (and biomass and fossil fuel burning), as well as abundant "crustal" PM silicate dust released from granitic ballast. Moreover, a higher concentration of PAHs was detected in the Barcelona subway system during the same sampling period. ${ }^{19}$ Hence, the observed situation would be reasonable, with some bacteria reported in polluted environments being predominating, as is the case of Methylobacterium. One source of origin for such dominant bacteria might be found on the subway air-ventilation system, including $A C$ ventilation inside trains. As previously reported, ${ }^{56,57}$ and references therein ${ }^{58}$; Methylobacterium-like bacteria have been found to be dominant in coil fin biofilms from air-handling (including air-conditioning) systems. Indeed, the desiccation resistance capacity observed for Methylobacterium was partly stated as an explanation of its dominance in such biofilms, generally subjected to extended periods of dehydration. Notwithstanding, the high relative abundances reported by this taxon are likely to be overestimated, as multiple rRNA operons (ie, five) have been reported previously from Methylobacterium genome sequences. ${ }^{59}$ Further studies to compare their abundance at indoor and outdoor systems should be carried out in order to determine whether this situation is idiosyncratic from the subway system. The other abundant and widespread taxa in the bacterial communities ( $>0.5 \%$ mean relative abundance and $>75 \%$ occurrence, Figure 5 panel B) have been described from diverse environmental sources, although mainly in soils. Some of them would thrive successfully on polluted environments as suggested by sphingomonads, ${ }^{60}$ besides their high metabolic diversity, as observed in members of the genus Paracoccus too. ${ }^{61}$ These have been found able to grow as methylotrophs and also as chemolithoautotrophs using products that may be related to vehicular and industrial emissions (eg, pollutants such as carbon monoxide or carbonyl sulfide), ${ }^{62,63}$ although given that Barcelona subway platforms have low CO levels, ${ }^{20}$ such an interpretation seems less applicable to our samples. Furthermore, members of Chitinophagaceae have been already described as airborne bacteria in urban environments. ${ }^{64}$

Samples from inside trains were further inspected to see whether the different $\mathrm{AC}$ settings (switch on or off) could infer some differences in terms of composition and structure. For instance, to see whether a shift on the proportion of bacterial types from different sources (human related vs. other environmental sources) exists. A number of exclusive taxa were found but no differences were observed for the dominant groups. Here, the effect that produces the opening and closing of doors and ventilation systems may help to explain the homogenization of results.

The degree of overlapping observed for airborne bacterial communities, which were dominated by a few widespread taxa, would seem to be more related to the prevalence of abiotic factors and bioburden on building structure (including trains) than for the commuters' occupancy. Thus, we hypothesize that this situation would be favored by the concentration of pollutants from outdoor air sources, nighttime maintenance activities, etc. (acting as facilitators), and moreover, biofouling in air-ventilation systems (including AC) might act as a possible source of such dominant taxa. Further investigations are needed to probe such hypothesis. Contrary to the observed results, one would expect a higher microbial diversity, expressed in terms of richness and ecological diversity, related to the intrinsic variety and also the quantity of commuters using the subway. Considering the low proportion of human-related microbiota in our sequence dataset, and that only one minor part of it can be related to potentially pathogenic bacteria, there is not an important biological exposure on any of the compartments, 
more than those related to the fact that this is a confined and crowded place where the transmission of respiratory diseases could be facilitated. Moreover, there was not a higher airborne concentration when the air-conditioning was switched off. A more detailed monitoring (yearly or interanual) would identify regularities and specific dynamics associated with the microbial communities and the bioburden found in subway systems. Studying seasonal variations would also be highly interesting, despite the relatively constant environmental conditions found in subway system.

\section{ACKNOWLEDGMENTS}

This study was supported by the Spanish Ministry of Economy and Competitiveness (MINECO) and FEDER funds (METRO CGL2012-33066, and BRIDGES CGL2015-69043-P), the European Union Seventh Framework Programme (FP7/2007-2013) for a Marie Curie ITN (Grant agreement no. 315760 HEXACOMM), and the IMPROVE LIFE Project (LIFE13 ENV/ES/000263). The authors would like to thank the Transports Metropolitans de Barcelona staff for their support during the sampling campaign. We would like to thank the editors and reviewers of the manuscript for their valuable corrections, suggestions, and encouraging comments.

\section{REFERENCES}

1. Barberan A, Henley J, Fierer N, Casamayor EO. Structure, inter-annual recurrence, and global-scale connectivity of airborne microbial communities. Sci Total Environ. 2014;487:187-195.

2. Hervas A, Camarero L, Reche I, Casamayor EO. Viability and potential for immigration of airborne bacteria from Africa that reach high mountain lakes in Europe. Environ Microbiol. 2009;11:1612-1623.

3. Douwes J, Thorne P, Pearce N, Heederik D. Bioaerosol health effects and exposure assessment: progress and prospects. Ann Occup Hyg. 2003;47:187-200.

4. Klepeis NE, Nelson WC, Ott WR, et al. The National Human Activity Pattern Survey (NHAPS): a resource for assessing exposure to environmental pollutants. J Expo Anal Environ Epidemiol. 2001;11:231-252.

5. Tringe SG, Zhang T, Liu X, et al. The airborne metagenome in an indoor urban environment. PLoS ONE. 2008;3:e1862.

6. Robertson CE, Baumgartner LK, Harris JK, et al. Culture-independent analysis of aerosol microbiology in a metropolitan subway system. Appl Environ Microbiol. 2013;79:3485-3493.

7. Nieuwenhuijsen MJ, Gómez-Perales JE, Colvile RN. Levels of particulate air pollution, its elemental composition, determinants and health effects in metro systems. Atmos Environ. 2007;41:7995-8006.

8. Dybwad M, Granum PE, Bruheim P, Blatny JM. Characterization of airborne bacteria at an underground subway station. Appl Environ Microbiol. 2012;78:1917-1929.

9. Leung MH, Wilkins D, Li EK, Kong FK, Lee PK. Indoor-air microbiome in an urban subway network: diversity and dynamics. Appl Environ Microbiol. 2014;80:6760-6770.

10. Hwang SH, Park JB. Comparison of culturable airborne bacteria and related environmental factors at underground subway stations between 2006 and 2013. Atmos Environ. 2014;84:289-293.

11. Rappé MS, Giovannoni SJ. The uncultured microbial majority. Annu Rev Microbiol. 2003;57:369-394.

12. Bogomolova E, Kirtsideli I. Airborne fungi in four stations of the St. Petersburg Underground railway system. Int Biodeterior Biodegradation. 2009;63:156-160.
13. Gilleberg SB, Faull JL, Graeme-Cook KA. A preliminary survey of aerial biocontaminants at six London Underground stations. Int Biodeterior Biodegradation. 1998;41:149-152.

14. Picco AM, Rodolfi M. Airborne fungi as biocontaminants at two Milan underground stations. Int Biodeterior Biodegradation. 2000;45:43-47.

15. Afshinnekoo E, Meydan C, Chowdhury S, et al. Geospatial resolution of human and bacterial diversity with city-scale metagenomics. Cell Syst. 2015;1:72-87.

16. Metasub International Consortium. The Metagenomics and Metadesign of the Subways and Urban Biomes (MetaSUB) international consortium inaugural meeting report. Microbiome. 2016;4: $1-14$.

17. Querol X, Moreno T, Karanasiou A, et al. Variability of levels and composition of PM10 and PM2.5 in the Barcelona metro system. Atmos Chem Phys. 2012;12:5055-5076.

18. Martins V, Moreno T, Minguillón MC, et al. Exposure to airborne particulate matter in the subway system. Sci Total Environ. 2015;511:711-722.

19. Martins V, Moreno T, Minguillón MC, et al. Origin of inorganic and organic components of PM2.5 in subway stations of Barcelona, Spain. Environ Pollut. 2016;208 Part A:125-136.

20. Moreno T, Pérez N, Reche C, et al. Subway platform air quality: assessing the influences of tunnel ventilation, train piston effect and station design. Atmos Environ. 2014;92:461-468.

21. Moreno T, Martins V, Querol X, et al. A new look at inhalable metalliferous airborne particles on rail subway platforms. Sci Total Environ. 2015;505:367-375.

22. Blais Lecours $P$, Veillette M, Marsolais D, Duchaine C. Characterization of bioaerosols from dairy barns: reconstructing the puzzle of occupational respiratory diseases by using molecular approaches. Appl Environ Microbiol. 2012;78:3242-3248.

23. Bonifait L, Veillette M, Létourneau V, Grenier D, Duchaine C. Detection of Streptococcus suis in bioaerosols of swine confinement buildings. Appl Environ Microbiol. 2014;80:3296-3304.

24. Bonifait L, Charlebois R, Vimont A, et al. Detection and quantification of airborne norovirus during outbreaks in healthcare facilities. Clin Infect Dis. 2015;61:299-304.

25. Verreault D, Gendron L, Rousseau GM, et al. Detection of airborne lactococcal bacteriophages in cheese manufacturing plants. Appl Environ Microbiol. 2011;77:491-497.

26. Comeau A, Li W, Tremblay J, Carmack E, Lovejoy C. Arctic Ocean microbial community structure before and after the 2007 record sea ice minimum. PLoS ONE. 2011;6:e27492.

27. Edgar RC. UPARSE: highly accurate OTU sequences from microbial amplicon reads. Nat Methods. 2013;10:996-998.

28. Caporaso JG, Kuczynski J, Stombaugh J, et al. QIIME allows analysis of high-throughput community sequencing data. Nat Methods. 2010;7:335-336.

29. Pruesse E, Peplies J, Glockner FO. SINA: accurate high-throughput multiple sequence alignment of ribosomal RNA genes. Bioinformatics. 2012;28:1823-1829.

30. Quast C, Pruesse E, Yilmaz P, et al. The SILVA ribosomal RNA gene database project: improved data processing and web-based tools. Nucleic Acids Res. 2013;41:D590-D596.

31. Oksanen J, Blanchet FG, Kindt R, et al. vegan: Community Ecology Package. 2013. R package version 2.0-10.

32. Legendre P, Gallagher ED. Ecologically meaningful transformations for ordination of species data. Oecologia. 2001;129:271-280.

33. Clarke KR, Warwick RM. Similarity-based testing for community pattern: the two-way layout with no replication. Mar Biol. 1994;118:167-176.

34. Schloss PD, Westcott SL, Ryabin T, et al. Introducing mothur: opensource, platform-independent, community-supported software for describing and comparing microbial communities. Appl Environ Microbiol. 2009;75:7537-7541. 
35. Human Microbiome Project Consortium. Structure, function and diversity of the healthy human microbiome. Nature. 2012;486:207-214.

36. Wickham H. ggplot2: Elegant Graphics for Data Analysis. New York: Springer-Verlag; 2009.

37. Ecker DJ, Sampath R, Willett P, et al. The Microbial Rosetta Stone Database: a compilation of global and emerging infectious microorganisms and bioterrorist threat agents. BMC Microbiol. 2005;5:1-17.

38. Ramette A. Multivariate analyses in microbial ecology. FEMS Microbiol Ecol. 2007;62:142-160.

39. Otto M. Staphylococcus epidermidis - the "accidental" pathogen. Nat Rev Microbiol. 2009;7:555-567.

40. Awad AHA. Environmental study in subway metro stations in Cairo, Egypt. J Occup Health. 2002;44:112-118.

41. Hwang SH, Yoon CS, Ryu KN, Paik SY, Cho JH. Assessment of airborne environmental bacteria and related factors in 25 underground railway stations in Seoul, Korea. Atmos Environ. 2010;44:1658-1662.

42. Rinsoz T, Duquenne P, Greff-Mirguet G, Oppliger A. Application of real-time PCR for total airborne bacterial assessment: comparison with epifluorescence microscopy and culture-dependent methods. Atmos Environ. 2008;42:6767-6774.

43. Amann RI, Wolfgang L, Schleifer K. Phylogenetic identification and in situ detection of individual microbial cells without cultivation. Microbiol Rev. 1995;59:143-169.

44. Letourneau V, Nehme B, Meriaux A, Masse D, Duchaine C. Impact of production systems on swine confinement buildings bioaerosols. J Occup Environ Hyg. 2009;7:94-102.

45. Nehme B, Létourneau V, Forster RJ, Veillette M, Duchaine C. Cultureindependent approach of the bacterial bioaerosol diversity in the standard swine confinement buildings, and assessment of the seasonal effect. Environ Microbiol. 2008;10:665-675.

46. Yang W, Elankumaran S, Marr LC. Concentrations and size distributions of airborne influenza A viruses measured indoors at a health centre, a day-care centre and on aeroplanes. $J R$ Soc Interface. 2011;8:1176-1184

47. Fabian P, Mcdevitt JJ, Dehaan WH, et al. Influenza virus in human exhaled breath: an observational study. PLoS ONE. 2008;3:e2691.

48. van Elden LJ, Nijhuis M, Schipper P, Schuurman R, van Loon AM. Simultaneous detection of influenza viruses $A$ and $B$ using real-time quantitative PCR. J Clin Microbiol. 2001;39:196-200.

49. Wei Z, McEvoy M, Razinkov V, et al. Biophysical characterization of influenza virus subpopulations using field flow fractionation and multiangle light scattering: correlation of particle counts, size distribution and infectivity. J Virol Methods. 2007;144:122-132.

50. PHAC. Rhinovirus - Pathogen safety data sheet - infectious substances - Public Health Agency of Canada. 2011.

51. Selvaraju SB, Selvarangan R. Evaluation of three Influenza A and $B$ real-time reverse transcription-PCR assays and a new 2009 H1N1 Assay for detection of Influenza viruses. J Clin Microbiol. 2010;48:3870-3875.
52. Latgé J-P. Aspergillus fumigatus and Aspergillosis. Clin Microbiol Rev. 1999;12:310-350.

53. Adams RI, Bhangar S, Pasut W, et al. Chamber bioaerosol study: outdoor air and human occupants as sources of indoor airborne microbes. PLoS ONE. 2015;10:e0128022.

54. Meadow JF, Altrichter AE, Kembel SW, et al. Indoor airborne bacterial communities are influenced by ventilation, occupancy, and outdoor air source. Indoor Air. 2014;24:41-48.

55. Green PN. Methylobacterium. In: Dworkin M, Falkow S, Rosenberg E, Schleifer K-H, Stackebrandt E, eds. The Prokaryotes - A Handbook on the Biology of Bacteria. Vol. 5. New York: Springer-Verlag; 2006:257-265.

56. Hugenholtz P, Cunningham MA, Hendrikz JK, Fuerst JA. Desiccation resistance of bacteria isolated from an air handling system biofilm determined using a simple quantitative membrane filter method. Lett Appl Microbiol. 1995;21:41-46.

57. Hugenholtz P, Fuerst JA. Heterotrophic bacteria in an air-handling system. Appl Environ Microbiol. 1992;58:3914-3920.

58. Simmons RB, Rose LJ, Crow SA, Ahearn DG. The occurrence and persistence of mixed biofilms in automobile air conditioning systems. Curr Microbiol. 1999;39:141-145.

59. Vuilleumier S, Chistoserdova L, Lee M-C, et al. Methylobacterium genome sequences: a reference blueprint to investigate microbial metabolism of C1 compounds from natural and industrial sources. PLoS ONE. 2009;4:e5584.

60. Balkwill DL, Fredrickson JK, Romine MF. Sphingomonas and related genera. In: Dworkin M, Falkow S, Rosenberg E, Schleifer K, Stackebrandt E, eds. The Prokaryotes - A Handbook on the Biology of Bacteria. Vol. 7. New York: Springer-Verlag; 2006:605-629.

61. Kelly DP, Rainey FA, Wood AP. The genus Paracoccus. In: Dworkin M, Falkow S, Rosenberg E, Schleifer K-H, Stackebrandt E, eds. The Prokaryotes - A Handbook on the Biology of Bacteria. Vol. 5. New York: Springer-Verlag; 2006:232-249.

62. Jordan LS, Mcdonald RI, Kraczkiewicz-Dowjat JA, et al. Autotrophic growth on carbon disulfide is a property of novel strains of Paracoccus denitrificans. Arch Microbiol. 1997;168:225-236.

63. Tolli JD, Sievert SM, Taylor CD. Unexpected diversity of bacteria capable of carbon monoxide oxidation in a coastal marine environment, and contribution of the Roseobacter-associated clade to total CO oxidation. Appl Environ Microbiol. 2006;72:1966-1973.

64. Kembel SW, Jones E, Kline J, et al. Architectural design influences the diversity and structure of the built environment microbiome. ISME J. 2012;6:1469-1479.

\section{SUPPORTING INFORMATION}

Additional Supporting Information may be found online in the supporting information tab for this article. 